\title{
CONFERENCE PARTICIPANTS
}

Abramowicz, Halina Andrianov, Alexander

Anselmino, Mauro

Anthonis, Tinne

Antonioli, Pietro

Arneodo, Michele

Aschenauer, Elke-Caroline

Babukhadia, Levan

Bacchetta, Alessandro

Badelek, Barbara

Bartels, Joachim

Basile, Maurizio

Bassi, Gabriele

Bastianelli, Fiorenzo

Beckmann, Marc

Bellagamba, Lorenzo

Bernstein, Robert

Bertolin, Alessandro

Bhadra, Sampa

Bianchi, Nicola

Bland, Leslie

Bluemlein, Johannes

Bodek, Arie

Boehrer, Armin

Boer, Daniel

Bojak, Ingo

Bonacorsi, Daniele

Bonsignori, Giovanni Carlo

Boscherini, Davide

Brown, Duncan

Brugnera, Riccardo

Bruni, Graziano

Bruni, Alessia

Buchmuller, Wilfried

Buschhorn, Gerd

Bussey, Peter J

Capitani, Gian Paolo

Capua, Marcella

Cashmore, Roger J.

Chakanau, Siarhei
Tel Aviv University

INFN - Bologna

University of Torino

University of Antwerp

INFN - Bologna

University of Piemonte Orientale and INFN

DESY - Zeuthen

State University of New York at Stony Brook

Vrije Universiteit

Uppsala University

Hamburg University

University of Bologna

University of Bologna

University of Bologna

DESY - Hamburg

INFN - Bologna

Fermilab

University of Padova

York University

INFN - Frascati

Indiana University

DESY

University of Rochester

Siegen University

RIKEN-BNL Research Center

CSSM, University of Adelaide

University of Bologna

University of Bologna

INFN - Bologna

DESY - Hamburg

University of Padova and INFN

INFN - Bologna

INFN - Bologna

DESY - Hamburg

MPI (Werner-Heisenberg-Institut)

University of Glasgow

INFN - Frascati

University of Calabria

CERN

Argonne National Laboratory 
Chekelian, Vladimir

Cifarelli, Luisa

Collard, Caroline

Collins, John

Contalbrigo, Marco

Cooper-sarkar, Amanda

Corradi, Massimo

Costa, Maria Jose

Dainton, John

D'Alesio, Umberto

Dasgupta, Mrinal

De Pasquale, Salvatore

De Roeck, Albert

Deshpande, Abhay

Devenish, Robin

Diaconu, Cristinel

Diehl, Markus

Di Nezza, Pasquale

Dodge, Gail

Dokshitzer, Yuri

Dubak, Ana

Eckstein, Doris

Eisenberg, Yehuda

Elsen, Eckhard

Enberg, Rikard

Ermolaev, Boris

Eskreys, Andrzej

Fabbri, Riccardo

Favart, Laurent

Ferron, Stephane

Fiore, Roberto

Fontannaz, Michel

Foster, Brian

Freund, Andreas

Fricke, Ulrich

Frixione, Stefano

Garfagnini, Alberto

Garutti, Erika

Gavillet, Philippe

Gehrmann, Thomas

Gieseke, Stefan

Golec-Biernat, Krzysztof

Golling, Tobias

Goncharov, Maxim
MPI (Werner-Heisenberg-Institut)

University of Salerno

Universite Libre de Bruxelles

Penn State University

University of Ferrara

Oxford University

INFN - Bologna

IFIC - Valencia

University of Liverpool

Cagliari University

DESY, Hamburg

INFN - Bologna

CERN

RIKEN-BNL Research Center

Oxford University

Centre de Physique des Particules de Marseille

DESY, Hamburg

INFN - Frascati

Old Dominion University

University Paris Sud

MPI (Werner-Heisenberg-Institut)

DESY - Zeuthen

Weizmann Institute

DESY - Hamburg

Uppsala University

Ioffe Physico-Technical Institute

Institute of Nuclear Physics

University of Ferrara

Universite Libre de Bruxelles

LPNHE - Paris

University of Calabria and INFN

Laboratoire de Physique Theorique

Bristol University

Regensburg University

DESY - Hamburg

INFN - Genova / Lapp - Annecy

Hamburg University

NIKHEF

CERN

CERN

Hamburg University

Hamburg University/INP Krakow

Kirchhoff-Institut f. Physik

Kansas State University 
Gonzalez, Oscar

Goulianos, Konstantin

Grazzini, Massimiliano

Grebenyuk, Oleg

Grindhammer, Günter

Guzey, Vadim

Haidt, Dieter

Heinsius, Fritz-Herbert

Helbing, Klaus

Hiller, Karlheinz

Hodgson, Paul

Iacobucci, Giuseppe

Iashvili, IashviliIa

Isenhower, Donald

Issever, Cigdem

Ivanov, Igor

Jakob, Rainer

Janssen, Xavier

Kcira, Dorian

Kienzle-Focacci, M. Novella

Kiesling, Christian

Klanner, Robert

Klasen, Michael

Klein, Max

Knies, Gerhard

Koike, Yuji

Kooijman, Paul

Korsch, Wolfgang

Korzhavina, Irina

Kotikov, Anatoly

Kowal, Agnieszka

Kreisel, Arik

Kroll, Peter

Kroseberg, Juergen

Kuhn, Sebastian E.

Kumano, Shunzo

Lastovicka, Tomas

Levy, Aharon

Limentani, Silvia

Lipatov, Lev N.

Long, Kenneth
Universidad Autonoma de Madrid

Rockefeller University

University of Florence

Petersburg Nuclear Physics Institute

MPI - Munich

CSSM, Adelaide University

DESY - Hamburg

Freiburg University

Erlangen University

DESY - Zeuthen

The University of Sheffield

INFN - Bologna

University of California, Riverside

Abilene Christian University

Dortmund University

IKP, Forschungszentrum Juelich

University of Wuppertal

Universite Libre de Bruxelles

University of Wisconsin

Geneva University

MPI

DESY - Hamburg

Hamburg University

DESY - Zeuthen

DESY - Hamburg

Niigata University

NIKHEF

University of Kentucky

Moscow State University

Joint Institute for Nuclear Reseach

University of Mining and Metallurgy -

Tel Aviv University

University of Wuppertal

University of Zurich

Old Dominion University

Saga University

IFH - DESY

Tel Aviv University

Univeristy of Padova and INFN

Russia Academy of Science

Imperial College London 
Longhin, Andrea

Lönnblad, Leif

Lopez-Duran Viani, Amaya

Lublinsky, Michael

Magnea, Lorenzo

Marage, Pierre

Margotti, Anselmo

Martin, John

Martin, Alan

Martinez, Mario

Matsushita, Takashi

Meyer, Joachim

Meyer, Andreas

Miller, C. Andrew

Miyama, Masanori

Mohrdieck, Susanne

Morfin, Jorge G.

Muccifora, Valeria

Mueller, Dieter

Mulders, Piet

Munier, Stephane

Murgia, Francesco

Nagano, Kunihiro

Nania, Rosario

Newman, Paul

Niebuhr, Carsten

Nisius, Richard

Nowak, Wolf-Dieter

Oganessyan, Karo

Olsson, Jan

Padley, B. Paul

Paganis, Efstathios

Palmonari, Federico

Papa, Alessandro

Patrizii, Laura

Pellegrino, Antonio

Peschanski, Robi

Peterson, Gerald

Petronzio, Roberto

Potenza, Renato

Potter, Bjorn

Radici, Marco
University of Padova

Lund University

DESY - Zeuthen

Technion-Israeli Institute of Technology

University of Torino

Universite Libre de Bruxelles

INFN - Bologna

University of Toronto

University of Durham

DESY - Hamburg

University of Oxford

DESY - Hamburg

Hamburg University

TRIUMF

Tokyo Metropolitan University

Hamburg University

Fermilab

INFN - Frascati

University of Wuppertal

Vrije Universiteit Amsterdam

INFN - Firenze

INFN - Cagliari

KEK - IPNS

INFN - Bologna

University of Birmingham

DESY - Hamburg

CERN

DESY - Zeuthen

INFN - Frascati

DESY - Hamburg

Rice University

Columbia University

University of Bologna

University of Calabria and INFN

INFN - Bologna

Argonne National Laboratory

Service de Physique Theorique (SPhT) of Saclay

University of Massachusetts

INFN - Roma

University of Catania

MPI - Munich

INFN - Pavia 
Ralston, John

Reisert, Burkard

Repond, Jose

Rizvi, Eram

Rondon, Oscar

Royon, Christophe

Ryckbosch, Dirk

Saito, Naohito

Salam, Gavin

Sartorelli, Gabriella

Schagen, Sven

Schildknecht, Dieter

Schilling, Frank-Peter

Schleper, Peter

Schoeffel, Laurent

Schoerner, Thomas

Selvi, Marco

Smalska, Barbara

Stamenov, Dimiter

Stancari, Michelle

Stanco, Luca

Stasto, Anna

Stella, Bruno R.

Stirling, W. James

Stoesslein, Uta

Stump, Daniel

Szczurek, ANTONI

Tassi, Enrico

Taylor, Russell

Teubner, Thomas

Thomas, Eric

Thorne, Robert

Tokushuku, Katsuo

Truoel, Peter

Turcato, Monica

Vacca, Gian Paolo

Van Mechelen, Pierre

Venturi, Giovanni

Vincter, Manuella

Vlachos, Sotirios

Vogt, Andreas

Volmer, Jochen
University of Kansas

MPI - Munich

Argonne National Laboratory

Birmingham University

University of Virginia

CEA Saclay - Brookhaven - UT Arlington

University of Gent

RIKEN - RIKEN BNL Research Center

CERN

University of Bologna

NIKHEF

University of Bielefeld

DESY - Hamburg

DESY - Hamburg

CEA - Saclay

MPI - Munich

University of Bologna

Warsaw University

Inst. for Nucl. Res. and Nucl. Energy - Sofia

INFN - Ferrara

INFN - Padova

INFN - Firenze

University of Rome I

University of Durham

University of Colorado

Michigan State University

Institute of Nuclear Physics

NIKHEF

University College London

RWTH - Aachen

INFN - Frascati

Cambridge University

KEK - IPNS

University of Zuerich

University of Padova

Hamburg University

University of Antwerpen (UIA)

University of Bologna

University of Alberta

University of Basel

Leiden University

DESY - Zeuthen 
Vorobiev, Igor

Wallny, Rainer

Weber, Ainas

Weiskopf, Christoph

Whitmore, Jim

Windmolders, Roland

Wobisch, Markus

Wolf, Guenter

Yamashita, Taro

Yamazaki, Yuji

Yoshida, Rik

Zarnecki, A. Filip

Zawiejski, Leszek

Zhang, Zhiqing

Zichichi, Antonino

Zotov, Nikolai

Zucchelli, Stefano
Carnegie Mellon University

University Zurich

Bonn University

University of Erlangen

Penn State University/DESY

Bonn University

DESY - Hamburg

DESY - Hamburg

KEK

KEK - IPNS

Argonne National Laboratory

Warsaw University

Institute of Nuclear Physics

LAL - Paris Sud University

University of Bologna

Moscow State University

University of Bologna 